\title{
B-SPLINE BASES AND OSCULATING FLATS: ONE RESULT OF H.-P. SEIDEL REVISITED
}

\author{
Marie-Laurence MaZure ${ }^{1}$
}

\begin{abstract}
Along with the classical requirements on B-splines bases (minimal support, positivity, normalization) we show that it is natural to introduce an additional "end point property". When dealing with multiple knots, this additional property is exactly the appropriate requirement to obtain the poles of nondegenerate splines as intersections of osculating flats at consecutive knots.
\end{abstract}

Mathematics Subject Classification. 65D17.

Received: May 13, 2002.

\section{INTRODUCTION}

In any spline space, the expression "B-spline basis" commonly denotes a normalized basis consisting of minimally supported functions which are positive on the interior of their supports. Such bases are of crucial importance in geometric design for, their properties ensure spline curves to be located in the convex hull of their control points, the influence of each control point being as local as possible.

In [2], Goodman established the existence of such bases in polynomial spline spaces of arbitrary degree with regular one-banded lower triangular totally positive connection matrices. The result was generalized to arbitrary regular lower triangular totally positive connection matrices by Dyn and Micchelli [1]. In [7,8] Seidel used the latter existence to prove the existence of blossoms [6] for geometrically continuous polynomial splines. The values of the blossoms were defined by means of intersections of osculating flats. His paper was a fundamental one, for the idea of using intersections of osculating flats originated the extension of the blossoming theory outside the polynomial framework, in particular by Pottmann [5] (see also [3]).

However, we recently noticed that, unless we limit ourselves to the case of simple knots, the proof of existence of blossoms given in [8] is not complete. This is due to the three classical axioms of B-spline bases (minimal support, positivity, normalization) being insufficient to achieve the result in the case of multiple knots: indeed, they do not guarantee the poles of nondegenerate splines to be obtained as intersections of osculating flats. Note that in the case of multiple knots, they do not guarantee unicity of such a basis either. These few remarks led us to wonder about the exact axioms to require on B-spline bases, and this is the object of the present paper.

The second section investigates the most general context of splines with sections in arbitrary spaces, and with connections defined by arbitrary lower triangular matrices, with positive diagonal elements. It contains various observations about minimally supported splines which justify the introduction of an additional requirement on

Keywords and phrases. Geometric design, B-spline basis, blossoming, osculating flats.

1 Laboratoire de Modélisation et Calcul (LMC-IMAG), Université Joseph Fourier, BP 53, 38041 Grenoble Cedex, France.

e-mail: mazure@imag.fr 
B-spline bases to make the difference between two consecutive B-splines the supports of which have the same left or right end point. A similar condition was actually used by Dyn and Micchelli to exhibit a B-spline basis in the polynomial case. In Section 3, we show that the additional axiom is exactly what is needed to enable us to define poles as intersections of a number of osculating flats at some knots. In particular, it is the finishing touch to complete the proof of Seidel's result.

\section{B-SPLINE BASES}

In order to guarantee the nice behaviour of a spline space for geometric design purposes, it is essential to have at our disposal a B-spline basis. However, what exactly do we mean by a B-spline basis? This is the question we shall address in the present section.

\subsection{Basic requirements on B-spline bases}

Throughout the paper, given a real number $x$ and a nonnegative integer $m$, the notation $x^{[m]}$ will stand for $x$ repeated $m$ times.

Consider a sequence of knots $\mathcal{T}=\left(t_{\ell}\right)_{\ell \in \mathbb{Z}}$, with $t_{\ell}<t_{\ell+1}$ and $\lim _{|l| \rightarrow+\infty}\left|t_{\ell}\right|=+\infty$. To each knot $t_{\ell}$, let us allocate a multiplicity $m_{\ell} \in\{0, \ldots, n\}$. We suppose that the corresponding knot vector $\mathcal{K}:=\left(t_{\ell}\left[m_{\ell}\right]\right)_{\ell \in \mathbb{Z}}$ is infinite, i.e. that infinitely many multiplicities are not equal to zero. When necessary we shall rewrite the knot vector as $\mathcal{K}=\left(\xi_{j}\right)_{j \in \mathbb{Z}}$, with $\xi_{j} \leq \xi_{j+1}$.

For each $\ell \in \mathbb{Z}$, we denote by $\mathcal{E}_{\ell}$ an $(n+1)$-dimensional linear subspace of $C^{n}\left(\left[t_{\ell}, t_{\ell+1}\right]\right)$ containing the constants, and by $\mathcal{M}_{\ell}$ a lower triangular matrix of order $n-m_{\ell}$ with positive diagonal. Associated with these data, we define the spline space $\mathcal{S}$ as the space of all continuous functions $S: \mathbb{R} \rightarrow \mathbb{R}$ the restrictions of which to $\left[t_{\ell}, t_{\ell+1}\right]$ belong to $\mathcal{E}_{\ell}$ for any $\ell \in \mathbb{Z}$, and which satisfy the connection conditions

$$
\left(S^{\prime}\left(t_{\ell}^{+}\right), \ldots, S^{\left(n-m_{\ell}\right)}\left(t_{\ell}^{+}\right)\right)^{T}=\mathcal{M}_{\ell} \cdot\left(S^{\prime}\left(t_{\ell}^{-}\right), \ldots, S^{\left(n-m_{\ell}\right)}\left(t_{\ell}^{-}\right)\right)^{T}, \quad \ell \in \mathbb{Z}
$$

In this most general context, a B-spline basis is commonly meant as a sequence of functions $\mathcal{N}_{\ell}, \ell \in \mathbb{Z}$, meeting the following classical requirements:

$(\mathrm{BSB})_{1}$ support condition: for all $\ell \in \mathbb{Z}$ the support of $\mathcal{N}_{\ell}$ is equal to $\left[\xi_{\ell}, \xi_{\ell+n+1}\right]$,

$(\mathrm{BSB})_{2}$ decomposition condition: for any $S \in \mathcal{S}^{d}, d \geq 1$, there exists a sequence $\left(T_{\ell}\right)_{\ell \in \mathbb{Z}}$ of points of $\mathbb{R}^{d}$ such that:

$$
S(t)=\sum_{\ell \in \mathbb{Z}} \mathcal{N}_{\ell}(t) T_{\ell}, \quad t \in \mathbb{R}
$$

$(\mathrm{BSB})_{3}$ positivity condition: for all $\ell \in \mathbb{Z}, \mathcal{N}_{\ell}$ is positive on the interior of its support, $(\mathrm{BSB})_{4}$ normalisation condition: $\sum_{\ell \in \mathbb{Z}} \mathcal{N}_{\ell}(t)=1$ for all $t \in \mathbb{R}$.

The knot vector $\mathcal{K}$ being infinite, for any $k \in \mathbb{Z}$, we can consider the greatest integer $q$ such that $\xi_{q} \leq t_{k}$. Throughout the paper, we shall denote it by $j(k)$. It is thus the only integer such that $\xi_{j(k)} \leq t_{k}, \xi_{j(k)+1} \geq t_{k+1}$, and, clearly, for all $q \geq 0, j(k+q)=j(k)+\sum_{i=k+1}^{k+q} m_{i}$.

Let us first state a few elementary consequences of the classical properties $(\mathrm{BSB})_{i}$.

Proposition 2.1. Assume the sequence $\mathcal{N}_{\ell}, \ell \in \mathbb{Z}$, to satisfy the two conditions $(\mathrm{BSB})_{1}$ and $(\mathrm{BSB})_{2}$. Then, each function $\mathcal{N}_{\ell}$ belongs to the space $\mathcal{S}$, and for any spline function $S: \mathbb{R} \rightarrow \mathbb{R}^{d}$, the decomposition (2.2) is unique. 
Proof. Given two integers $k, s$, with $s \geq 0$, denote by $\mathcal{S}_{k, s}$ the restriction of $\mathcal{S}$ to $\left[t_{k}, t_{k+s+1}\right]$. Each space $\mathcal{E}_{\ell}$ being $(n+1)$-dimensional, due to the connection conditions (2.1), the space $\mathcal{S}_{k, s}$ is of dimension at least

$$
(n+1)(s+1)-\sum_{i=k+1}^{k+s}\left(n-m_{i}+1\right)=n+1+\sum_{i=k+1}^{k+s} m_{i} .
$$

On account of the support condition (BSB) $)_{1}$, for $t \in\left[t_{k}, t_{k+s+1}\right]$, the sum in (2.2) reduces to the indices $\ell$ such that $j(k)-n \leq \ell \leq j(k)+\sum_{i=k+1}^{k+s} m_{i}$. We can thus conclude that the space $\mathcal{S}_{k, s}$ is of exact dimension $n+1+\sum_{i=k+1}^{k+s} m_{i}$, and that the restrictions of the functions $\mathcal{N}_{\ell}, j(k)-n \leq \ell \leq j(k)+\sum_{i=k+1}^{k+s} m_{i}$, form a basis of $\mathcal{S}_{k, s}$. This observation readily proves the unicity of any decomposition (2.2).

On the other hand, for a given $\ell \in \mathbb{Z}$, we can choose $k, s$ so that $t_{k}<\xi_{\ell}$ and $t_{k+s+1}>\xi_{\ell+n+1}$. We know that, restricted to $\left[t_{k}, t_{k+s+1}\right]$, the function $\mathcal{N}_{\ell}$ belongs to the space $\mathcal{S}_{k, s}$. Its support being contained in $] t_{k}, t_{k+s+1}[$, the function $\mathcal{N}_{\ell}$ is thus an element of the space $\mathcal{S}$.

As a consequence, as long as the two conditions $(\mathrm{BSB})_{1}$ and $(\mathrm{BSB})_{2}$ hold, conversely, any sequence $T_{\ell}, \ell \in \mathbb{Z}$, of points in $\mathbb{R}^{d}$, provides an element $S \in \mathcal{S}^{d}$, defined by means of equality (2.2). We shall call the points $T_{\ell}$ the poles of $S$.

Given $S \in \mathcal{S}^{d}$, for any $\ell \in \mathbb{Z}$, the affine flat spanned by the set $\left\{S(x), x \in\left[t_{\ell}, t_{\ell+1}\right]\right\}$ is of dimension at most $n$ since $\mathcal{E}_{\ell}$ is $(n+1)$-dimensional. We say that the spline $S$ is nondegenerate when this affine flat is of dimension $n$ whatever the integer $\ell$. Nondegenerate splines can be recognized from their poles as stated in the following result.

Proposition 2.2. Assume the sequence $\mathcal{N}_{\ell}, \ell \in \mathbb{Z}$, to satisfy the three conditions $(\mathrm{BSB})_{1},(\mathrm{BSB})_{2}$ and $(\mathrm{BSB})_{4}$. Then, the spline $S$ provided by equality $(2.2)$ is nondegenerate iff, for all integer $k \in \mathbb{Z}$, the $(n+1)$ points $T_{j(k)-n}, \ldots, T_{j(k)}$ are affinely independent.

Proof. On account of the three conditions $(\mathrm{BSB})_{1},(\mathrm{BSB})_{2}$ and $(\mathrm{BSB})_{4}$, we can write:

$$
S(x)=\sum_{\ell=j(k)-n}^{j(k)} \mathcal{N}_{\ell}(x) T_{\ell}, \quad \sum_{\ell=j(k)-n}^{j(k)} \mathcal{N}_{\ell}(x)=1, \quad x \in\left[t_{k}, t_{k+1}\right] .
$$

Therefore,

$$
\left\{S(x), x \in\left[t_{k}, t_{k+1}\right]\right\} \subset \text { aff }\left(T_{j(k)-n}, \ldots, T_{j(k)}\right) .
$$

If $S$ is nondegenerate, then the left-hand side of (2.4) spans an $n$-dimensional affine space and the inclusion (2.4) is thus an equality, which proves the affine independence of the $n+1$ points $T_{j(k)-n}, \ldots, T_{j(k)}$. The converse part follows from the fact that the functions $\mathcal{N}_{\ell}, j(k)-n \leq \ell \leq j(k)$, form a basis of the space $\mathcal{S}_{k, 1}$ (see the proof of Prop. 2.1). Indeed, this enables us to find $n+1$ points $x_{0}, \ldots, x_{n} \in\left[t_{k}, t_{k+1}\right]$ so that the matrix $\left(\mathcal{N}_{j(k)-i}\left(x_{q}\right)\right)_{0 \leq i, q \leq n}$ is regular. Due to the affine independence of the points $T_{j(k)-n}, \ldots, T_{j(k)}$, the corresponding points $S\left(x_{0}\right), \ldots, S\left(x_{n}\right)$ are then affinely independent, which proves the affine space spanned by the set $\left\{S(x), x \in\left[t_{k}, t_{k+1}\right]\right\}$ to be $n$-dimensional.

\subsection{Nondegenerate splines and osculating flats}

The restriction of a spline $S \in \mathcal{S}^{d}$ to any interval $\left[t_{\ell}, t_{\ell+1}\right]$ being $C^{n}$, we can consider its osculating flat of order $i \leq n$ at any point $x \in \mathbb{R}$, namely the affine flat $\operatorname{Osc}_{i} S(x)$, passing through the point $S(x)$ and the direction of which is spanned by the first $i$ derivatives of $S$ at $x$. At a knot $t_{\ell}$, clearly we have to consider both the left and right osculating flats $\operatorname{Osc}_{i} S\left(t_{\ell}^{-}\right)$and $\operatorname{Osc}_{i} S\left(t_{\ell}^{+}\right)$defined using the left and right derivatives 
respectively. However, for $i \leq n-m_{\ell}$, the connection condition (2.1) and our requirement on the matrix $\mathcal{M}_{\ell}$ guarantee that $\mathrm{Osc}_{i} S\left(t_{\ell}^{-}\right)=\operatorname{Osc}_{i} S\left(t_{\ell}^{+}\right)$, and we shall simply denote it by $\operatorname{Osc}_{i} S\left(t_{\ell}\right)$.

Once and for all, we select a nondegenerate spline $S \in \mathcal{S}^{d}$. For any $x \in \mathbb{R}$ and any $k \leq n$, the dimension of the (possibly left or right) osculating flat $\operatorname{Osc}_{k} S(x)$ does not depend on $S$. Indeed, if $\widetilde{S}$ denotes another nondegenerate spline, then $\mathrm{Osc}_{k} \widetilde{S}(x)$ is the image of $\mathrm{Osc}_{k} S(x)$ under an injective affine map. Hence, in particular the following two properties:

(H) for all $\ell \in \mathbb{Z}$, the osculating flat $\operatorname{Osc}_{n-m_{\ell}} S\left(t_{\ell}\right)$ is of dimension $\left(n-m_{\ell}\right)$,

$(\mathrm{H})^{\prime}$ for all $\ell \in \mathbb{Z}$, such that $m_{\ell} \neq 0$, the osculating flat $\operatorname{Osc}_{n-m_{\ell}} S\left(t_{\ell}\right)$ is of dimension $\left(n-m_{\ell}\right)$,

are independent of the chosen nondegenerate spline $S$. Note that $(\mathrm{H})$ is satisfied as soon as each $\mathcal{E}_{\ell}$ is what we call a $W$-space on $\left[t_{\ell}, t_{\ell+1}\right]$, that is, if the Wronskian of a basis of $\mathcal{E}_{\ell}$ does not vanish on $\left[t_{\ell}, t_{\ell+1}\right]$. As a particular example, it holds when each $\mathcal{E}_{\ell}$ is the restriction to $\left[t_{\ell}, t_{\ell+1}\right]$ of the polynomial space $\mathcal{P}_{n}$ of degree $n$. As for the weaker condition $(\mathrm{H})^{\prime}$, it is satisfied in particular when each $\mathcal{E}_{\ell}$ is a what we call a quasi $W$-space on $\left[t_{\ell}, t_{\ell+1}\right]$ : this means that, at any point $x \in\left[t_{\ell}, t_{\ell+1}\right]$, the determinant obtained by replacing, in the Wronskian of a basis, all $n$th derivatives by the derivatives of some order $r(x)$ is not equal to zero (see [4]).

Proposition 2.3. Assume the sequence $\mathcal{N}_{\ell}, \ell \in \mathbb{Z}$, to satisfy the three conditions $(\mathrm{BSB})_{1},(\mathrm{BSB})_{2}$ and $(\mathrm{BSB})_{4}$, and denote by $T_{\ell}, \ell \in \mathbb{Z}$, the poles of the nondegenerate spline $S$. Let us consider integers $\ell \in \mathbb{Z}, s \in \mathbb{N}$, such that $p:=n-\sum_{i=\ell+1}^{\ell+s} m_{i} \geq 0$. If condition $(\mathrm{H})$ is satisfied, then we have:

$$
\bigcap_{i=\ell+1}^{\ell+s} \operatorname{Osc}_{n-m_{i}} S\left(t_{i}\right)=\operatorname{aff}\left(T_{j(\ell)-p}, \ldots, T_{j(\ell)}\right)
$$

Proof. Suppose that $m_{k} \neq 0$. Since $\xi_{\ell}=t_{k}$ iff $j(k)-m_{k}+1 \leq \ell \leq j(k)$, exactly $m_{k}$ functions $\mathcal{N}_{\ell}$ have $t_{k}$ as the left end point of their support, namely those for which $j(k)-m_{k}+1 \leq \ell \leq j(k)$. Equality (2.3) thus gives:

$$
S\left(t_{k}\right)=\sum_{\ell=j(k)-n}^{j(k)-m_{k}} \mathcal{N}_{\ell}\left(t_{k}\right) T_{\ell}, \quad \sum_{\ell=j(k)-n}^{j(k)-m_{k}} \mathcal{N}_{\ell}\left(t_{k}\right)=1 .
$$

According to Proposition 2.1, the $m_{k}$ functions $\mathcal{N}_{\ell}, j(k)-m_{k}+1 \leq \ell \leq j(k)$, belong to $\mathcal{S}$. Consequently, they all vanish at least $n-m_{k}+1$ times at the point $t_{k}$. From (2.3) we can thus derive:

$$
S^{(i)}\left(t_{k}^{+}\right)=\sum_{\ell=j(k)-n}^{j(k)-m_{k}} \mathcal{N}_{\ell}^{(i)}\left(t_{k}^{+}\right) T_{\ell}, \quad \sum_{\ell=j(k)-n}^{j(k)-m_{k}} \mathcal{N}_{\ell}^{(i)}\left(t_{k}^{+}\right)=0, \quad 1 \leq i \leq n-m_{k} .
$$

Note that relations (2.6) and (2.7) still hold if $m_{k}=0$. From (2.6) and (2.7) we can deduce that:

$$
\mathrm{Osc}_{n-m_{k}} S\left(t_{k}\right)=\operatorname{Osc}_{n-m_{k}} S\left(t_{k}^{+}\right) \subset \operatorname{aff}\left(T_{j(k)-n}, \ldots, T_{j(k)-m_{k}}\right) .
$$

The assumption (H) allows us to conclude that $\mathrm{Osc}_{n-m_{k}} S\left(t_{k}\right)=$ aff $\left(T_{j(k)-n}, \ldots, T_{j(k)-m_{k}}\right)$. Since, for all $k \in \mathbb{Z}$, $j(k)-m_{k}=j(k-1)$, this is nothing but equality (2.5) corresponding to $s=1$. Given $s \geq 1$, suppose that $p:=n-\sum_{i=\ell+1}^{\ell+s} m_{i} \geq m_{\ell+s+1}$, and that equality (2.5) is satisfied. We can then write:

$$
\begin{aligned}
\bigcap_{i=\ell+1}^{\ell+s+1} \operatorname{Osc}_{n-m_{i}} S\left(t_{i}\right) & =\operatorname{aff}\left(T_{j(\ell)-p}, \ldots, T_{j(\ell)}\right) \cap \operatorname{aff}\left(T_{j(\ell+s)+m_{\ell+s+1}-n}, \ldots, T_{j(\ell+s)}\right), \\
& =\operatorname{aff}\left(T_{j(\ell+s)-n}, \ldots, T_{j(\ell)}\right) \cap \operatorname{aff}\left(T_{j(\ell+s)-n+m_{\ell+s+1}}, \ldots, T_{j(\ell+s)}\right) .
\end{aligned}
$$


The spline $S$ being nondegenerate, the $(n+1)$ points $T_{j(\ell+s)-n}, \ldots, T_{j(\ell+s)}$ are affinely independent. Hence, the latter equality gives:

$$
\bigcap_{i=\ell+1}^{\ell+s+1} \operatorname{Osc}_{n-m_{i}} S\left(t_{i}\right)=\operatorname{aff}\left(T_{j(\ell+s)-n+m_{\ell+s+1}}, \ldots, T_{j(\ell)}\right),
$$

which is the equality (2.5) obtained when replacing $s$ by $s+1$.

With the same kind of arguments, we can also state the following result.

Proposition 2.3bis. With the same assumptions as in Proposition 2.3, if condition $(\mathrm{H})^{\prime}$ is satisfied, then

$$
\bigcap_{\substack{i=\ell+1 \\ m_{i} \neq 0}}^{\ell+s} \operatorname{Osc}_{n-m_{i}} S\left(t_{i}\right)=\operatorname{aff}\left(T_{j(\ell)-p}, \ldots, T_{j(\ell)}\right) .
$$

Let us mention the following interesting particular case of the previous results.

Corollary 2.4. Under the same assumptions as in Proposition 2.3, for any integers $\ell \in \mathbb{Z}, s \in \mathbb{N}$, such that $\sum_{i=\ell+1}^{\ell+s} m_{i}=n$, if $(\mathrm{H})^{\prime}$ is satisfied, we have:

$$
\left\{T_{j(\ell)}\right\}=\bigcap_{\substack{i=\ell+1 \\ m_{i} \neq 0}}^{\ell+s} \operatorname{Osc}_{n-m_{i}} S\left(t_{i}\right)
$$

and the condition $m_{i} \neq 0$ can be omitted in (2.10) under assumption $(\mathrm{H})$.

Remark 2.5. If all knots are simple, that is if $m_{\ell}=1$ for all $\ell \in \mathbb{Z}$, the previous result states that, under assumption $(\mathrm{H})$, all poles of a nondegenerate spline are obtained by intersecting osculating hyperplanes at $n$ consecutive knots. Indeed, taking $\xi_{\ell}:=t_{\ell}$ for all $\ell,(2.10)$ yields:

$$
\left\{T_{\ell}\right\}=\bigcap_{i=\ell+1}^{\ell+n} \operatorname{Osc}_{n-1} S\left(t_{i}\right), \quad \ell \in \mathbb{Z}
$$

In contrast, if any multiplicities are allowed, the equality:

$$
\left(\xi_{q+1}, \ldots, \xi_{q+n}\right)=\left(t_{\ell+1}^{\left[m_{\ell+1}\right]}, \ldots, t_{\ell+s}^{\left[m_{\ell+s}\right]}\right)
$$

is reached only by exception. Therefore the classical properties of B-splines are not sufficient to ensure that all poles are obtained as intersections of a number of osculating flats at consecutive knots. This suggests the necessity of introducing additional axioms for B-splines to guarantee such a result.

Still, our arguments to prove (2.10) are similar to those used by Seidel in [8] to deduce from the existence of B-spline bases in geometrically continuous polynomial spline spaces (proved in [1] by Dyn and Micchelli) that in such spline spaces all poles could be constructed by intersecting osculating flats. Unfortunately, for his proof to be valid, he did need the equality (2.12) to be satisfied, and he probably did not notice the exceptional feature of such an equality.

\subsection{What is a B-spline basis?}

With the same assumptions as in Proposition 2.1, suppose that all multiplicities are equal to 1 . Consider a spline $S \in \mathcal{S}^{d}$, given by (2.2), the support of which is contained in the interval $\left[t_{k}, t_{k+s+1}\right]$. From arguments similar to those used in the proof of Proposition 2.1, we can deduce that $T_{i}=0$ for all $i \leq k-1$ and for all $i \geq$ 
$k+s+1-n$. In particular, applying this with $s=n$ proves that, up to a multiplicative constant, the function $\mathcal{N}_{k}$ is the only function in $\mathcal{S}$ the support of which is equal $\left[t_{k}, t_{k+n+1}\right]$. Therefore there exists at most one normalized sequence of functions satisfying $(\mathrm{BSB})_{1}$ and $(\mathrm{BSB})_{2}$. On the opposite, if any multiplicities are allowed, the support condition $\operatorname{Supp}\left(\mathcal{N}_{k}\right)=\left[\xi_{k}, \xi_{k+n+1}\right]$ no longer characterizes the function $\mathcal{N}_{k}$ up to a multiplicative constant since it may even happen that $\left[\xi_{k}, \xi_{k+n+1}\right]=\left[\xi_{k^{\prime}}, \xi_{k^{\prime}+n+1}\right]$ for $k \neq k^{\prime}$. Another observation of the same nature: if the sequence $\mathcal{N}_{\ell}, \ell \in \mathbb{Z}$, satisfies the support, decomposition, and normalization conditions $(\mathrm{BSB})_{1},(\mathrm{BSB})_{2}$, and $(\mathrm{BSB})_{4}$ respectively, and if $\xi_{q}=\xi_{q+1}$, it is possible to replace the two functions $\mathcal{N}_{q}, \mathcal{N}_{q+1}$ respectively by $\alpha \mathcal{N}_{q}$ and $(1-\alpha) \mathcal{N}_{q}+\mathcal{N}_{q+1}$, where $\left.\alpha \in\right] 0,1[$, without altering any of the three properties above. This does not alter the positivity condition either. Hence, as soon as there exists at least one multiple knot, the existence of a sequence $\mathcal{N}_{\ell}, \ell \in \mathbb{Z}$, satisfying $(\mathrm{BSB})_{\mathrm{i}}, 1 \leq i \leq 4$, there actually exist infinitely many such sequences. This confirms the relevance of introducing additional axioms on the functions $\mathcal{N}_{\ell}$ so as to make up for this inconvenience.

Consider once more the case of simple knots. The support condition then makes it obvious that, for any $\ell$, as $x$ grows from $-\infty$ to $+\infty$, the quantity $\mathcal{N}_{\ell}(x)$ leaves the $x$-axis "quicker" than the quantity $\mathcal{N}_{\ell+1}(x)$ and also returns to it "quicker". We can formulate this as follows:

$$
\lim _{x \rightarrow t_{\ell}^{+}} \frac{\mathcal{N}_{\ell+1}(x)}{\mathcal{N}_{\ell}(x)}=\lim _{x \rightarrow t_{\ell+n+1}^{-}} \frac{\mathcal{N}_{\ell-1}(x)}{\mathcal{N}_{\ell}(x)}=0, \quad \ell \in \mathbb{Z}
$$

In the general case, we shall still require each $\mathcal{N}_{\ell}$ to leave the $x$-axis "quicker" than $\mathcal{N}_{\ell+1}$ and to return to it "quicker" too, in the sense that:

Definition 2.6. We say that a sequence $\mathcal{N}_{\ell}, \ell \in \mathbb{Z}$, is a B-spline basis if it satisfies the four properties (BSB) $1 \leq i \leq 4$, along with the following one:

$(\mathrm{BSB})_{5}$ end point condition: for all $\ell \in \mathbb{Z}$,

$$
\lim _{x \rightarrow \xi_{\ell}^{+}} \frac{\mathcal{N}_{\ell+1}(x)}{\mathcal{N}_{\ell}(x)}=0, \quad \lim _{x \rightarrow \xi_{\ell+n+1}^{-}} \frac{\mathcal{N}_{\ell-1}(x)}{\mathcal{N}_{\ell}(x)}=0 .
$$

This additional end point property enables us to make the difference between two consecutive B-splines and it guarantees the unicity of a possible B-spline basis.

Proposition 2.7. There exists at most one B-spline basis in the spline space $\mathcal{S}$.

Proof. Assume that the two sequences $\mathcal{N}_{\ell}, \mathcal{L}_{\ell}, \ell \in \mathbb{Z}$ satisfy the properties $(\mathrm{BSB})_{1}$, (BSB) $)_{2}$, and $(\mathrm{BSB})_{5}$. For all $\ell$ let us write $\mathcal{L}_{\ell}$ as

$$
\mathcal{L}_{\ell}(x)=\sum_{q \in \mathbb{Z}} a_{q}^{\ell} \mathcal{N}_{q}(x), \quad x \in \mathbb{R}
$$

Let $k$ be an integer such that $m_{k} \neq 0$. The support of $\mathcal{L}_{\ell}$ is contained in $\left[t_{k},+\infty\left[\right.\right.$ iff $\ell \geq j(k)-m_{k}+1$. Considering the restriction of $\mathcal{L}_{\ell}$ to any interval $\left[t_{r}, t_{r+1}\right]$, with $r<k$, it follows that $a_{q}^{\ell}=0$ for all $\ell \geq j(k)-m_{k}+1$ and all $q \leq j(k)-m_{k}$. For $j(k)-m_{k}+1 \leq \ell \leq j(k)$, we thus have:

$$
\mathcal{L}_{\ell}(x)=\sum_{q=j(k)-m_{k}+1}^{j(k)} a_{q}^{\ell} \mathcal{N}_{q}(x), \quad x \in\left[t_{k}, t_{k+1}\right] .
$$

From $(\mathrm{BSB})_{5}$ we can deduce that

$$
\lim _{x \rightarrow t_{k}^{+}} \frac{\mathcal{N}_{\ell}(x)}{\mathcal{N}_{j(k)-m_{k}+1}(x)}=\lim _{x \rightarrow t_{k}^{+}} \frac{\mathcal{L}_{\ell}(x)}{\mathcal{L}_{j(k)-m_{k}+1}(x)}=0, \quad \ell>j(k)-m_{k}+1 .
$$


On the other hand, for $x \in\left[t_{k}, t_{k+1}\right]$, and $j(k)-m_{k}+2 \leq \ell \leq j(k),(2.14)$ yields:

$$
\frac{\mathcal{L}_{\ell}(x)}{\mathcal{L}_{j(k)-m_{k}+1}(x)}=\frac{a_{j(k)-m_{k}+1}^{\ell}+\sum_{q=j(k)-m_{k}+2}^{j(k)} a_{q}^{\ell} \frac{\mathcal{N}_{q}(x)}{a_{j(k)-m_{k}+1}(x)}}{a_{j(k)-m_{k}+1}^{j(k)-m_{k}+1}+\sum_{q=j(k)-m_{k}+2}^{j(k)} a_{q}^{j(k)-m_{k}+1} \frac{\mathcal{N}_{q}(x)}{\mathcal{N}_{j(k)-m_{k}+1}(x)}} .
$$

Taking (2.15) into account, the latter equality yields:

$$
a_{j(k)-m_{k}+1}^{\ell}=0, \quad j(k)-m_{k}+2 \leq \ell \leq j(k) .
$$

Considering successively $\lim _{x \rightarrow t_{k}^{+}} \mathcal{L}_{\ell}(x) / \mathcal{L}_{j(k)-m_{k}+2}(x)$ for $j(k)-m_{k}+2 \leq \ell \leq j(k)$, then for $j(k)-m_{k}+3 \leq$ $\ell \leq j(k), \lim _{x \rightarrow t_{k}^{+}} \mathcal{L}_{\ell}(x) / \mathcal{L}_{j(k)-m_{k}+3}(x)$, and so forth, we eventually obtain that $a_{q}^{\ell}=0$ for $j(k)-m_{k}+1 \leq q<$ $\ell \leq j(k)$. Hence, we have proved that $a_{q}^{\ell}=0$ for all $q, \ell \in \mathbb{Z}, q<\ell$. Similarly, using the right part of (2.13) it will be possible to prove that $a_{q}^{\ell}=0$ for all $q, \ell \in \mathbb{Z}, q>\ell$. Hence $\mathcal{L}_{\ell}=a_{\ell}^{\ell} \mathcal{N}_{\ell}$. If the two sequences also satisfy $(\mathrm{BSB})_{4}$, the unicity of the decomposition of the constant function $\mathbb{I}$ proves that $a_{\ell}^{\ell}=1$ for all $\ell \in \mathbb{Z}$.

\section{Obtaining POLES AS INTERSECTIONS OF OSCULATING FLATS}

In this section we shall prove that the additional end point property is exactly what is needed to obtain all poles of a nondegenerate spline as intersections of osculating flats at convenient knots.

Any $n$-tuple $\left(\xi_{q+1}, \ldots, \xi_{q+n}\right)$ composed of consecutive elements of the knot vector $\mathcal{K}$ can always be written as follows:

$$
\left(\xi_{q+1}, \ldots, \xi_{q+n}\right)=\left(t_{\ell}^{[\alpha]}, t_{\ell+1}^{\left[m_{\ell+1}\right]}, \ldots, t_{\ell+s}^{\left[m_{\ell+s}\right]}, t_{\ell+s+1}^{[\beta]}\right)
$$

with $m_{\ell} \neq 0, m_{\ell+s+1} \neq 0,0 \leq \alpha<m_{\ell}, 0 \leq \beta<m_{\ell+s+1}$.

Theorem 3.1. Let $\mathcal{N}_{\ell}, \ell \in \mathbb{Z}$, be a sequence of functions satisfying the conditions $(\mathrm{BSB})_{\mathrm{i}}, 1 \leq i \leq 4$, and let $S \in \mathcal{S}^{d}$ be a nondegenerate spline, with poles $T_{\ell}, \ell \in \mathbb{Z}$. Then the following four properties are equivalent:

(i) All poles of the spline $S$ are obtained as intersections of osculating flats, as follows: if the $n$-tuple $\left(\xi_{q+1}, \ldots, \xi_{q+n}\right)$ is given by (3.1), then the corresponding pole $T_{q}$ satisfies:

$$
\left\{T_{q}\right\}=\operatorname{Osc}_{n-\alpha} S\left(t_{\ell}^{+}\right) \cap \bigcap_{i=\ell+1}^{\ell+s} \operatorname{Osc}_{n-m_{i}} S\left(t_{i}\right) \cap \operatorname{Osc}_{n-\beta} S\left(t_{\ell+s+1}^{-}\right) .
$$

(ii) For any $\ell \in \mathbb{Z}$, and any $\alpha, 0 \leq \alpha \leq m_{\ell}$,

$$
\begin{aligned}
& \operatorname{Osc}_{n-\alpha} S\left(t_{\ell}^{-}\right)=\operatorname{aff}\left(T_{j(\ell-1)-n+\alpha}, \ldots, T_{j(\ell-1)}\right), \\
& \operatorname{Osc}_{n-\alpha} S\left(t_{\ell}^{+}\right)=\operatorname{aff}\left(T_{j(\ell)-n}, \ldots, T_{j(\ell)-\alpha}\right) .
\end{aligned}
$$

(iii) Condition $(\mathrm{H})$ of Section 2 is satisfied, and, given $\ell \in \mathbb{Z}$, if $\xi_{\ell}=t_{k}$ and $\xi_{\ell+n+1}=t_{k^{\prime}}$, the function $\mathcal{N}_{\ell}$ vanishes exactly $n-m_{k}+p+1$ at $t_{k}^{+}$and exactly $n-m_{k^{\prime}}+p^{\prime}+1$ at $t_{k^{\prime}}^{-}$, where

$$
p:=\sharp\left\{q<\ell \mid \xi_{q}=t_{k}\right\}, \quad p^{\prime}:=\sharp\left\{q>\ell+n+1 \mid \xi_{q}=t_{k^{\prime}}\right\} .
$$

(iv) For all $\ell \in \mathbb{Z}$, the left and right osculating flats $\operatorname{Osc}_{n} S\left(t_{\ell}^{-}\right)$and $\operatorname{Osc}_{n} S\left(t_{\ell}^{+}\right)$are $n$-dimensional, and the sequence $\mathcal{N}_{q}, q \in \mathbb{Z}$, satisfies the end point condition (BSB) 5 , i.e. it is a B-spline basis. 
Proof.

$\bullet$ (i) $\Rightarrow$ (ii). By Proposition 2.2, we know that, for all $\ell \in \mathbb{Z}$, the $n+1$ points $T_{j(\ell)-n}, \ldots, T_{j(\ell)}$ are affinely independent.

According to (3.2), for any integer $q$ such that $j(\ell)-n \leq q \leq j(\ell)-m_{\ell}$ (that is, for any $q$ such that the $n$-tuple $\left(\xi_{q+1}, \ldots, \xi_{q+n}\right)$ contains $\left.t_{\ell}{ }^{\left[m_{\ell}\right]}\right)$, the point $T_{q}$ belongs to $\mathrm{Osc}_{n-m_{\ell}} S\left(t_{\ell}\right)$. From the linear independence of the $\left(n-m_{\ell}+1\right)$ points $T_{j(\ell)-n}, \ldots, T_{j(\ell)-m_{\ell}}$, we can thus conclude that

$$
\mathrm{Osc}_{n-m_{\ell}} S\left(t_{\ell}\right)=\operatorname{aff}\left(T_{j(\ell)-n}, \ldots, T_{j(\ell)-m_{\ell}}\right)
$$

Now, (3.2) also implies that $T_{j(\ell)-\alpha} \in \mathrm{Osc}_{n-\alpha} S\left(t_{\ell}^{+}\right)$and $T_{j(\ell-1)-n+\alpha} \in \mathrm{Osc}_{n-\alpha} S\left(t_{\ell}^{-}\right)$for $0 \leq \alpha<m_{\ell}$. Hence, for $0 \leq \alpha<m_{\ell}, \operatorname{Osc}_{n-\alpha} S\left(t_{\ell}^{+}\right)$contains $\operatorname{Osc}_{n-m_{\ell}} S\left(t_{\ell}\right)$ and the points $T_{j(\ell)-m_{\ell}+1}, \ldots, T_{j(\ell)-\alpha}$, while Osc ${ }_{n-\alpha} S\left(t_{\ell}^{-}\right)$ contains $\mathrm{Osc}_{n-m_{\ell}} S\left(t_{\ell}\right)$ and the points $T_{j(\ell-1)-n+\alpha}, \ldots, T_{j(\ell-1)-n+m_{\ell-1}}=T_{j(\ell)-n-1}$. Again the affine independence of the points $T_{j(\ell)-n}, \ldots, T_{j(\ell)}$ on the one hand, and of the points $T_{j(\ell-1)-n}, \ldots, T_{j(\ell-1)}=T_{j(\ell)-m_{\ell}}$ on the other hand lead to (3.4) and (3.3).

$\bullet$ (ii) $\Rightarrow$ (iii). For any $\ell \in \mathbb{Z}$, the equality $\operatorname{Osc}_{n-m_{\ell}} S\left(t_{\ell}\right)=\operatorname{aff}\left(T_{j(\ell)-n}, \ldots, T_{j(\ell)-m_{\ell}}\right)$ and the linear independence of the points $T_{j(\ell)-n}, \ldots, T_{j(\ell)}$ proves that $\mathrm{Osc}_{n-m_{\ell}} S\left(t_{\ell}\right)$ is of dimension $\left(n-m_{\ell}\right)$. Hence condition (H) of Section 2 is satisfied.

Consider an integer $k$ such that $m_{k} \neq 0$. As already observed, the $m_{k}$ functions $\mathcal{N}_{\ell}, j(k)-m_{k}+1 \leq \ell \leq j(k)$, have $t_{k}$ as the left end point of their support. Similarly, exactly $m_{k}$ functions $\mathcal{N}_{\ell}$ have $t_{k}$ as the right end point of their support, namely those for which $j(k)-n-m_{k} \leq \ell \leq j(k)-n-1$. In order to prove (iii) we thus have to show that the following equalities hold:

$$
\begin{gathered}
\mathcal{N}_{j(k)-m_{k}+r}^{(i)}\left(t_{k}^{+}\right)=0,0 \leq i \leq n-m_{k}+r-1, \mathcal{N}_{j(k)-m_{k}+r}^{\left(n-m_{k}+r\right)}\left(t_{k}^{+}\right) \neq 0,1 \leq r \leq m_{k}, \\
\mathcal{N}_{j(k)-n-r}{ }^{(i)}\left(t_{k}^{-}\right)=0,0 \leq i \leq n-m_{k}+r-1, \mathcal{N}_{j(k)-n-r}^{\left(n-m_{k}+r\right)}\left(t_{k}^{-}\right) \neq 0,1 \leq r \leq m_{k} .
\end{gathered}
$$

Taking into account the affine independence of the points $T_{j(k)-n}, \ldots, T_{j(k)}$, equality (3.4) implies that, for $1 \leq r \leq m_{k}$, the two osculating flats $\mathrm{Osc}_{n-m_{k}+r} S\left(t_{k}^{+}\right)$and $\mathrm{Osc}_{n-m_{k}+r-1} S\left(t_{k}^{+}\right)$are not equal. Therefore, the point $S\left(t_{k}\right)+S^{\left(n-m_{k}+r\right)}\left(t_{k}^{+}\right)$belongs to aff $\left(T_{j(k)-n}, \ldots, T_{j(k)-m_{k}+r}\right)$, but it does not belong to aff $\left(T_{j(k)-n}, \ldots\right.$, $\left.T_{j(k)-m_{k}+r-1}\right)$. Now, equality (2.6) is still valid, and by differentiation of (2.3) at $t_{k}^{+}$up to the order $n-m_{k}+r$, $1 \leq r \leq m_{k}$, we obtain:

$$
S^{\left(n-m_{k}+r\right)}\left(t_{k}^{+}\right)=\sum_{\ell=j(k)-n}^{j(k)} \mathcal{N}_{\ell}^{\left(n-m_{k}+r\right)}\left(t_{k}^{+}\right) T_{\ell}, \quad \sum_{\ell=j(k)-n}^{j(k)} \mathcal{N}_{\ell}^{\left(n-m_{k}+r\right)}\left(t_{k}^{+}\right)=0
$$

Therefore, due to the affine independence of the points $T_{j(k)-n}, \ldots, T_{j(k)}$, the fact that $S\left(t_{k}\right)+S^{\left(n-m_{k}+r\right)}\left(t_{k}^{+}\right)$ belongs to $\operatorname{aff}\left(T_{j(k)-n}, \ldots, T_{j(k)-m_{k}+r}\right) \backslash \operatorname{aff}\left(T_{j(k)-n}, \ldots, T_{j(k)-m_{k}+r-1}\right)$ implies that:

$$
\mathcal{N}_{q}^{\left(n-m_{k}+r\right)}\left(t_{k}^{+}\right)=0, \quad j(k)-m_{k}+r+1 \leq q \leq j(k), \quad \mathcal{N}_{j(k)-m_{k}+r}{ }^{\left(n-m_{k}+r\right)}\left(t_{k}^{+}\right) \neq 0,
$$

which is nothing but (3.6). Taking into account the affine independence of the points $T_{j(k)-m_{k}-n}, \ldots, T_{j(k)-m_{k}}$, conditions (3.7) follow in a similar way from (2.6), and from the equality

$$
S^{\left(n-m_{k}+r\right)}\left(t_{k}^{-}\right)=\sum_{q=j(k)-m_{k}-n}^{j(k)-m_{k}} \mathcal{N}_{q}^{\left(n-m_{k}+r\right)}\left(t_{k}^{-}\right) T_{k}, \quad \sum_{q=j(k)-m_{k}-n}^{j(k)-m_{k}} \mathcal{N}_{q}^{\left(n-m_{k}+r\right)}\left(t_{k}^{-}\right)=0 .
$$

-(iii) $\Rightarrow$ (ii). From (iii) it is easy to derive that the $n$ right derivatives $S^{\prime}\left(t_{\ell}^{+}\right), \ldots, S^{(n)}\left(t_{\ell}^{+}\right)$are linearly independent. Hence, for $0 \leq \alpha \leq m_{\ell}$, the osculating flat $\operatorname{Osc}_{n-\alpha} S\left(t_{\ell}^{+}\right)$is of dimension $n-\alpha$. Taking into account 
the fact that, for $j(\ell)-m_{\ell}+1 \leq q \leq j(\ell)$ all functions $\mathcal{N}_{q}$ vanish $n-m_{\ell}+1$ times at the knot $t_{\ell}$, we can also deduce from (iii) the inclusion $\operatorname{Osc}_{n-\alpha} S\left(t_{\ell}^{+}\right) \subset \operatorname{aff}\left(T_{j(\ell)-n}, \ldots, T_{j(\ell)-\alpha}\right)$, which is thus an equality. Similar arguments work for $\operatorname{Osc}_{n-\alpha} S\left(t_{\ell}^{-}\right)$.

-(iii) $\Rightarrow$ (iv). As seen in the proof of (iii) $\Rightarrow$ (ii), (iii) implies that the osculating flat $\mathrm{Osc}_{n} S\left(t_{\ell}^{+}\right)$is $n$-dimensional. Moreover condition (iii) also clearly implies (2.13) by using Taylor expansions at the knot $t_{\ell}$ of consecutive functions $\mathcal{N}_{q}$ having this knot as the left or right end point of their support.

$\bullet$ (iv) $\Rightarrow$ (iii). The space $\mathcal{E}_{\ell}$ is composed of $C^{n}$ functions on $\left[t_{\ell}, t_{\ell+1}\right]$, and we know that $\mathcal{N}_{j(\ell)-m_{\ell}+1}$ vanishes at least $n-m_{\ell}+1$ times at $t_{\ell}$. By induction, the left part of $(2.12)$ implies that, for $1 \leq r \leq m_{\ell}$, the function $\mathcal{N}_{j(\ell)-m_{\ell}+r}$ vanishes at least $n-m_{\ell}+r$ times at $t_{\ell}$. Taking additionally into account the linear independence of the first $n$ derivatives at $t_{\ell}^{+}$proves that it cannot vanish more than $n-m_{\ell}+r$ times at $t_{\ell}$. Using the right equality in (2.12), similar arguments can be used to prove (3.7).

-(ii) $\Rightarrow$ (i). From (3.3) or (3.4) applied with $\alpha=m_{\ell}$, we can derive in particular that condition (H) of the previous section is satisfied. Therefore, since $n-\sum_{i=\ell+1}^{\ell+s} m_{i}=\alpha+\beta$, the corresponding equality (2.5) can be written as follows:

$$
\bigcap_{i=\ell+1}^{\ell+s} \operatorname{Osc}_{n-m_{i}} S\left(t_{i}\right)=\operatorname{aff}\left(T_{q-\beta}, \ldots, T_{q+\alpha}\right) .
$$

On the other hand, here equality (3.4) gives:

$$
\operatorname{Osc}_{n-\alpha} S\left(t_{\ell}^{+}\right)=\operatorname{aff}\left(T_{j(\ell)-n}, \ldots, T_{q}\right)=\operatorname{aff}\left(T_{q-n+\alpha}, \ldots, T_{q}\right) .
$$

The points $T_{j(\ell)-n}, \ldots, T_{j(\ell)}$ are affinely independent. The indices of all points $T_{r}$ involved in the right-hand sides of (3.8) and (3.9) satisfy $j(\ell)-n \leq r \leq q+\alpha=j(\ell)$. We thus have:

$$
\mathrm{Osc}_{n-\alpha} S\left(t_{\ell}^{+}\right) \cap \bigcap_{i=\ell+1}^{\ell+s} \operatorname{Osc}_{n-m_{i}} S\left(t_{i}\right)=\operatorname{aff}\left(T_{q-\beta}, \ldots, T_{q}\right)
$$

Similarly, from (3.3) we obtain:

$$
\operatorname{Osc}_{n-\beta} S\left(t_{\ell+s+1}^{-}\right)=\operatorname{aff}\left(T_{q}, \ldots, T_{q+n-\beta}\right) .
$$

The affine independence of the points $T_{j(\ell+s)-n}, \ldots, T_{j(\ell+s)}$ and the fact that the indices of all points $T_{r}$ involved in the right-hand sides of (3.10) and (3.11) satisfy $j(\ell+s)-n=q-\beta \leq r \leq j(\ell+s)=q+n-\beta$, eventually lead to (3.2).

Note that we can also express the condition on the zeros of each $\mathcal{N}_{\ell}$ stated in (iii) by saying that $\mathcal{N}_{\ell}$ is exactly $C^{n-m_{k}+p}$ at $\xi_{\ell}$ and exactly $C^{n-m_{k^{\prime}}+p^{\prime}}$ at $\xi_{\ell+n+1}$. On the other hand, observe that we did not make any use of the positivity condition $(\mathrm{BSB})_{3}$ in the proof of Theorem 3.1. This condition allows us to make the statements (3.6) and (3.7) more precise. For all integer $k$ such that $m_{k} \neq 0$, we actually have:

$$
\mathcal{N}_{j(k)-m_{k}+r}^{\left(n-m_{k}+r\right)}\left(t_{k}^{+}\right)>0, \quad(-1)^{n-m_{k}+r} \mathcal{N}_{j(k)-n-r}^{\left(n-m_{k}+r\right)}\left(t_{k}^{-}\right)>0, \quad 1 \leq r \leq m_{k}
$$

When writing the $n$-tuple $\left(\xi_{q+1}, \ldots, \xi_{q+n}\right)$ as in $(3.1)$, among the knots $t_{\ell+1}, \ldots, t_{\ell+s}$, those which have a zero multiplicity do not appear in the sequence $\xi_{q+1}, \ldots, \xi_{q+n}$. Hence it can be considered artificial to take them into account in the equality (3.2). This observation is also valid for $t_{\ell}{ }_{\ell}^{[\alpha]}$ when $\alpha=0$, and for $t_{\ell+s+1}{ }^{[\beta]}$ when $\beta=0$. For this very reason, we shall still write $\left(\xi_{q+1}, \ldots, \xi_{q+n}\right)$ as in $(3.1)$, but we shall now assume that $1 \leq \alpha \leq m_{\ell}$, $1 \leq \beta \leq m_{\ell+s+1}$. This is always possible provided that we allow the integer $s$ to be greater than or equal to -1 . 
By slightly adapting the arguments used in the previous proof, and in particular by using Proposition 2.3bis instead of Proposition 2.3, one can obtain the following result.

Theorem 3.1bis. Let $\mathcal{N}_{\ell}, \ell \in \mathbb{Z}$, be a sequence of functions satisfying the conditions (BSB) $)_{\mathrm{i}}, 1 \leq i \leq 4$, and let $S \in \mathcal{S}^{d}$ be a nondegenerate spline, with poles $T_{\ell}, \ell \in \mathbb{Z}$. Then the following four properties are equivalent:

(i) All poles of the spline $S$ are obtained as intersections of osculating flats, as follows: if (3.1) is satisfied, with $1 \leq \alpha \leq m_{\ell}, 1 \leq \beta \leq m_{\ell+s+1}$, and $s \geq-1$, then the pole $T_{q}$ satisfies:

$$
\left\{T_{q}\right\}=\operatorname{Osc}_{n-\alpha} S\left(t_{\ell}^{+}\right) \cap \bigcap_{\substack{i=\ell+1 \\ m_{i} \neq 0}}^{\ell+s} \operatorname{Osc}_{n-m_{i}} S\left(t_{i}\right) \cap \operatorname{Osc}_{n-\beta} S\left(t_{\ell+s+1}^{-}\right) .
$$

(ii) For any $\ell \in \mathbb{Z}$, and any $\alpha, 1 \leq \alpha \leq m_{\ell}$,

$$
\begin{aligned}
& \operatorname{Osc}_{n-\alpha} S\left(t_{\ell}^{-}\right)=\operatorname{aff}\left(T_{j(\ell-1)-n+\alpha}, \ldots, T_{j(\ell-1)}\right), \\
& \operatorname{Osc}_{n-\alpha} S\left(t_{\ell}^{+}\right)=\operatorname{aff}\left(T_{j(\ell)-n}, \ldots, T_{j(\ell)-\alpha}\right) .
\end{aligned}
$$

(iii) Condition $(\mathrm{H})^{\prime}$ of Section 2 is satisfied, and, if $\xi_{\ell}=t_{k}$ and $\xi_{\ell+n+1}=t_{k^{\prime}}$, and if $p$ and $p^{\prime}$ are defined as in (3.5), then the function $\mathcal{N}_{\ell}$ vanishes exactly (resp. at least) $n-m_{k}+p+1$ at $t_{k}$ if $p<m_{k}-1$ (resp. if $p=m_{k}-1$ ), and it vanishes exactly (resp. at least) $n-m_{k^{\prime}}+p^{\prime}+1$ at $t_{k^{\prime}}$ if $p^{\prime}<m_{k^{\prime}}-1$ (resp. if $\left.p^{\prime}=m_{k^{\prime}}-1\right)$,

(iv) For any integer $\ell \in \mathbb{Z}$ such that $m_{\ell} \neq 0$, the right and left osculating flats $\operatorname{Osc}_{n-1} S\left(t_{\ell}^{-}\right)$and $\operatorname{Osc}_{n-1} S\left(t_{\ell}^{+}\right)$ are $(n-1)$-dimensional, and the sequence $\mathcal{N}_{q}, q \in \mathbb{Z}$, satisfies the end point condition (BSB) $)_{5}$, i.e. it is a B-spline basis.

In [1], Dyn and Micchelli considered the case of polynomial splines with connection matrices on a closed bounded interval. When all connection matrices are totally positive (i.e. all their minors are nonnegative), they constructed a basis which satisfied the support, positivity, and normalization conditions [1] (Th. 4.6), and this is the result used by Seidel in $[7,8]$ to prove that all poles of what he called a universal spline (a particular nondegenerate spline) were obtained as intersections of osculating flats as in (3.2). Actually, in order to construct their basis, in their proof, the authors of [1] did require it to satisfy the conditions on zeros stated in (iii) of our Theorem 3.1. According to the latter proposition, this validates Seidel's result.

More generally, if for any $\ell \in \mathbb{Z}$, the space $\mathcal{E}_{\ell}$ is a $\mathrm{W}$-space on $\left[t_{\ell}, t_{\ell+1}\right]$ (see Sect. 2.1), existence of a B-spline basis in the space $\mathcal{S}$ automatically implies that all poles of a nondegenerate spline are given by (3.2). Using Theorem 3.1, existence of B-spline bases in a given W-spline space $\mathcal{S}$ and also in any spline space obtained from $\mathcal{S}$ by knot insertion, implies existence of blossoms in the space $\mathcal{S}$, and in particular it therefore implies that each $\mathcal{E}_{\ell}$ is an extended Chebyshev space on $\left[t_{\ell}, t_{\ell+1}\right]$. Actually, we shall prove in another paper that the converse property holds too, which will mean that existence of blossoms and existence of B-spline bases are equivalent in the context of W-splines. Using Theorem 3.1bis instead of Theorem 3.1 the result can be extended to the more general context of quasi-W-splines (see [4]).

\section{REFERENCES}

[1] N. Dyn and C.A. Micchelli, Piecewise polynomial spaces and geometric continuity of curves. Numer. Math. 54 (1988) 319-337.

[2] T.N.T. Goodman, Properties of $\beta$-splines. J. Approx. Theory 44 (1985) 132-153.

[3] M.-L. Mazure, Blossoming: a geometrical approach. Constr. Approx. 15 (1999) 33-68.

[4] M.-L. Mazure, Quasi-Chebyshev splines with connexion matrices. Application to variable degree polynomial splines. Comput. Aided Geom. Design 18 (2001) 287-298.

[5] H. Pottmann, The geometry of Tchebycheffian splines. Comput. Aided Geom. Design 10 (1993) 181-210.

[6] L. Ramshaw, Blossoms are polar forms. Comput. Aided Geom. Design 6 (1989) 323-358.

[7] H.-P. Seidel, New algorithms and techniques for computing with geometrically continuous spline curves of arbitrary degree. RAIRO Modél. Math. Anal. Numér. 26 (1992) 149-176.

[8] H.-P. Seidel, Polar forms for geometrically continuous spline curves of arbitrary degree. ACM Trans. Graphics 12 (1993) 1-34. 